\title{
Long-term memory for pictures and sentences
}

\author{
KERRY STOPHER and KIM KIRSNER \\ University of Western Australia, Nedlands 6009, Western Australia
}

\begin{abstract}
Two experiments were conducted to examine the fan effect in recognition memory for pictures and sentences. Subjects in the first experiment memorized sets of pictures $(\mathrm{N}=13)$ or sentences $(\mathrm{N}=13)$ in which the study items comprised concept combinations (e.g., the clock is on the television) that could be unambiguously depicted in each modality. The fan effect, contrasting study items involving unique- and shared-concepts combinations, was observed in the recognition reaction time data for sentences but not for pictures. Subjects in the second experiment $(N=17)$ memorized sets of pictures and sentences in which, in addition to the unique and shared intramodality conditions used in Experiment 1, subjects memorized items in each modality that shared concepts with items in the alternative modality. Although the fan effect was reduced for sentences in the second experiment, the intramodality results were qualitatively comparable to those obtained in Experiment 1. The results in the intermodality conditions indicated that, although pictorial study items influenced reaction time to sentences with which they shared a concept, the reverse was not the case. The results were thought to be inconsistent with the view that pictures and sentences enjoy a common representational format in longterm memory.
\end{abstract}

A question of current theoretical interest in cognitive psychology concerns the manner in which information about sentences and pictures is represented in memory. The central question is whether information resulting from the presumably distinct peripheral processes applied to sentences and pictures enjoys a common representational format in long-term memory. Paivio (1976), for example, has advanced the view that there are distinct imaginal and verbal memory stores that are specialized for the retention of pictorial and verbal or linguistic information, respectively. Anderson and Bower (1973), on the other hand, have proposed that information about pictures and sentences is retained in a common, propositional format. Their propositional model of human associative memory (HAM) has received extensive support from studies in which sentences have been used as stimuli (Anderson, 1974; Anderson \& Hastie, 1974; Lewis \& Anderson, 1976); however, little direct evidence has been reported from studies using pictorial stimuli. An important issue, therefore, and the one to which the present paper is directed, involves the question of whether or not Anderson and Bower's model can account for retention of pictorial as well as verbal information.

According to Anderson and Bower's (1973) model, when a sentence is presented for acquisition or recogni-

This research was reported by K. Stopher in an unpublished honors thesis, The propositional storage of pictures and sentences in long-term memory, University of Western Australia, 1977. The authors are grateful to F. I. M. Craik, L. Harris, M. C. Smith, and J. Winkelman for helpful comments on earlier versions of this paper. Requests for reprints should be addressed to Kim Kirsner, Department of Psychology, University of Western Australia, Perth, Nedlands 6009, Western Australia. tion, rules stored in long-term memory are applied by a linguistic parser to decompose the stimulus into appropriate components in working memory. The product of this parsing operation takes the form of a binary tree (see Figure 1) that consists of a number of abstract concept nodes in binary relationships to each other. The terminal units in each tree are attached to general concept nodes in long-term memory. When a recognition decision is required, appropriate searches are initiated simultaneously at each general concept node in the parsed sentence. As this search sequence is assumed to be both serial and self-terminating, reaction time will reflect the number of propositions attached to the relevant general concept node. When this model is applied to the pictorial domain, a perceptual, as distinct from linguistic, parser operates during the decomposi-

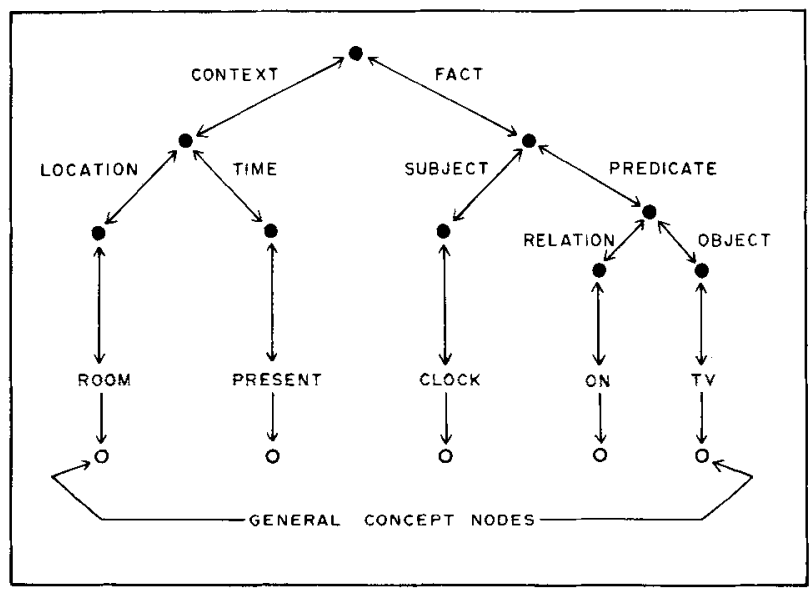

Figure 1. A binary propositional tree (adapted from Anderson \& Bower, 1973, p. 139). 
tion stage, but in other respects the model is insensitive to stimulus class. Thus, both the products of the two parsing processes and the subsequent retrieval operations applied to the underlying propositional representation are assumed to be at least qualitatively similar. If this is the case, then it follows that picture and sentence recognition should be sensitive to the same variables. In particular, reaction time to classify items as "old" or "new" should reflect the number of propositions attached to a given concept mode.

Anderson and Paulson (1978) examined this question using Identikit faces. In two studies, they found a fan effect reflecting the number of professions assigned to selected facial features during learning. In the learning phase of Anderson and Paulson's experiments, subjects were required to associate professional titles (e.g., "lawyer") with composite faces, and the fan manipulation was achieved by varying the number of labeled faces that included a particular example of a concept. The experiments described here differed in two respects from those reported by Anderson and Paulson. First, the training and test procedures were designed to avoid the use of verbal responses. A recognition memory task was used, knowledge of results was given, and a series of acquisition and test blocks were interleaved so that performance could be assessed under conditions in which verbal responses were not required in conjunction with either the verbal or the pictorial stimuli. The second difference concerned the nature of the concepts to be used. These were chosen so that (1) the same concept would be depicted in each modality and (2) the examples in each concept set would not be mutually exclusive. The issue of exclusivity is an important one. While it is, of course, possible to present a number of different pairs of eyes with one mouth under Identikit conditions, this does not occur in normal experience; each mouth can have only one pair of eyes associated with it. In the experiments reported here, the relationship between the concept classes is not constrained in this way. It would be quite common to experience more than one of the "items" in conjunction with a given "object." For example, a table could have a flower and a jug on it at the same time.

\section{EXPERIMENT 1}

Experiment 1 is a modified version of an experiment reported by Anderson (1974). Anderson taught subjects 26 simple sentences of the form, "A 'person' is in the "location," in which specific items were substituted for the general terms, "person" and "location," as in the sentence, "A hippie is in the park." The sentences were considered to express relationships that subjects were unlikely to have learned before. Two independent variables were manipulated: the number of propositions per person and the number of propositions per location. Any specific person or location contained in a sentence could be unique to that sentence, or it could be found in one or two other sentences. Subjects studied the sentences at a self-paced rate and were questioned to ascertain whether they knew every sentence. They were then randomly presented the sentences they had learned, together with an equal number of novel sentences constructed by recombining person and location concepts. Subjects were required to press one button if the sentence was previously learned and another button if it was novel.

Experiment 1 retained the essential features of the design used by Anderson (1974); however, as the element combinations were to be presented in pictures as well as sentences, several modifications were required. First, in order to avoid verbal questioning in the learning phase, a recognition memory procedure including knowledge or results was used throughout the experiment. Verbal questioning was thought to be inappropriate, as it might encourage subjects to use a verbal coding strategy. Second, in order to ensure an adequate level of accuracy, the task was divided into three phases involving learning, testing, and feedback, respectively, and this sequence was repeated eight times. Data analysis was then restricted to blocks of trials on which subjects achieved an overall accuracy level of $85 \%$ correct. Finally, as it was essential to select sentences that could be depicted unambiguously in pictorial form, these were chosen to express simple, familiar relationships. Thus the experiment should also demonstrate whether or not the fan effect is obtained when familiar objects and relationships are used.

\section{Method}

Design. The three factors included in the design were modality (sentences vs. pictures), repetition status ("old" vs. "new"), and, nested in the "old" condition, concept status (unique vs. shared concepts). Modality was a between-subjects variable, and 13 undergraduates acted as subjects in each group.

Each session consisted of eight blocks of trials, and each block included 16 new stimuli and the fixed set of 16 old stimuli. Eight of the old stimuli were drawn from the uniqueconcepts condition and shared no elements (items or objects) with any other old stimuli. The other eight old stimuli were drawn from the shared-concepts condition, in which each concept in every stimulus was shared with one other stimulus. The new stimuli in each block were randomly constructed from the remaining concept combinations.

Stimuli. The stimuli were constructed by combining examples from two sets of concepts. One set was termed "items," and the other set was termed "objects." The two sets are listed in Table 1. The concepts were familiar to subjects and could be unambiguously depicted in sentences or in schematic diagrams. The items were combined with the objects to express the relationship "The item is on the object." A total of 225 possible relationships could be formed. As an example, Figure 2 depicts the relationship between the concepts "clock" and "television" in each modality.

Sentences. All sentences were formed from letters measuring approximately $6 \mathrm{~mm}^{2}$. There was one initial uppercase letter, followed by lowercase letters. Each sentence consisted of six words of the form "The item is on the object." The words "The," "is," "on," and "the" were constant in every sentence. 
Table 1

Item Concepts and Object Concepts Used to Form Stimulus Relationships

\begin{tabular}{cc}
\hline Item Concepts & Object Concepts \\
\hline apple & bed \\
bird & boat \\
bottle & bucket \\
candle & car \\
cat & chair \\
clock & guitar \\
cup & hat \\
dog & ladder \\
flag & piano \\
flower & pram \\
jug & stair \\
key & stool \\
letter & table \\
pear & television \\
shoe & wall \\
\hline
\end{tabular}

Different word pairs from the concept lists were substituted for the item and object concepts.

The first four words of every sentence were placed from left to right on an imaginary horizontal line just above the central horizontal axis on the oscilloscope. The last two words were similarly placed on a horizontal line just below the central axis. As the total number of letters in different sentences varied, each sentence was adjusted so that the first and last letters of each word line were the same distance from each end of the oscilloscope screen. All letters in a sentence were displayed simultaneously.

Pictures. The pictures consisted of a schematic diagram of an item concept placed above a schematic diagram of an object concept so that the base of the former appeared to horizontally contact some part of the latter in a manner that most naturally expressed the relationship "The item is on the object." The individual item and object diagrams were plotted to preserve relative ordinal differences in size. Thus all items were smaller than the objects on which they were placed. The pictures ranged in size from $30 \times 50 \mathrm{~mm}$ to $70 \times 60 \mathrm{~mm}$. All pictures consisted of a number of lines plotted as points of light, as shown in Figure 2.

Selection. The stimulus selection procedure was designed to ensure that: (1) each session under a given stimulus class involved presentation of a unique subset of target stimuli from the item and object ensembles and (2) the same subsets of target stimuli were used in the picture and sentence conditions. Before each session, a program randomly selected four items and objects for the shared-concepts condition and eight items and objects for the unique-concepts condition from the item and object ensembles. The item and object concepts in the shared condition were then combined into eight item-object relationships, so that any item was related to two different objects and any object related to two different items. The items and objects in the unique condition were combined into eight unique relationships. Novel relationships were formed from all possible combinations of the items and objects except for those already selected for the target subsets. Items designated to represent the selected concept relationships were then accessed from files containing the 225 possible sets of coordinates for each of the stimulus class conditions.

Procedure. The experiment consisted of two phases.

Learning phase. The videomonitor instructed subjects that they would be presented 16 stimuli and would be required to concentrate on learning the relationship between the two components of each stimulus. This was followed by a consecutive display of the 16 stimuli on the oscilloscope. The eight stimuli in the shared-concepts condition were categorized into four pairs of stimuli, each of which included a common item concept. These were presented before the eight unique-concepts stimuli. Each stimulus appeared for $4.0 \mathrm{sec}$, followed by an interstimulus interval of $1.0 \mathrm{sec}$ before presentation of the next stimulus. This procedure was repeated three times.

Test phase. The entire test phase described below was repeated eight times for each subject.

Part 1: Trial Block-The videomonitor instructed subjects that the test phase was commencing and reminded them that they should respond as quickly as possible while achieving a block score of at least $85 \%$ correct. Subjects were required to press a "yes" button or a "no" button with the index finger of their dominant hand for old and new stimuli, respectively. Feedback was given on the oscilloscope ("yes" and "no" for correct and incorrect responses, respectively) after each trial. Each stimulus was presented for $1.5 \mathrm{sec}$ and followed, after feedback, by a 2.5 -sec intertrial interval.

Part 2: Block Feedback-When 32 responses were collected from each subject, the percentage of correct responses and the mean response latencies were displayed on the videomonitor for each subject for $20 \mathrm{sec}$. During this time, the experimenter discussed the results with the subjects and encouraged them to concentrate on responding as quickly as they could while reaching the $85 \%$ correct criterion.

Part 3: Refreshment Learning-The videomonitor instructed subjects that they would be presented the original 16 stimuli they had learned, in the same order, to refresh their memory. These stimuli were then presented once in the same manner as in the learning phase.

Apparatus. All stimulus selection, randomization, data acquisition, and feedback procedures were controlled by a PDP-11/10

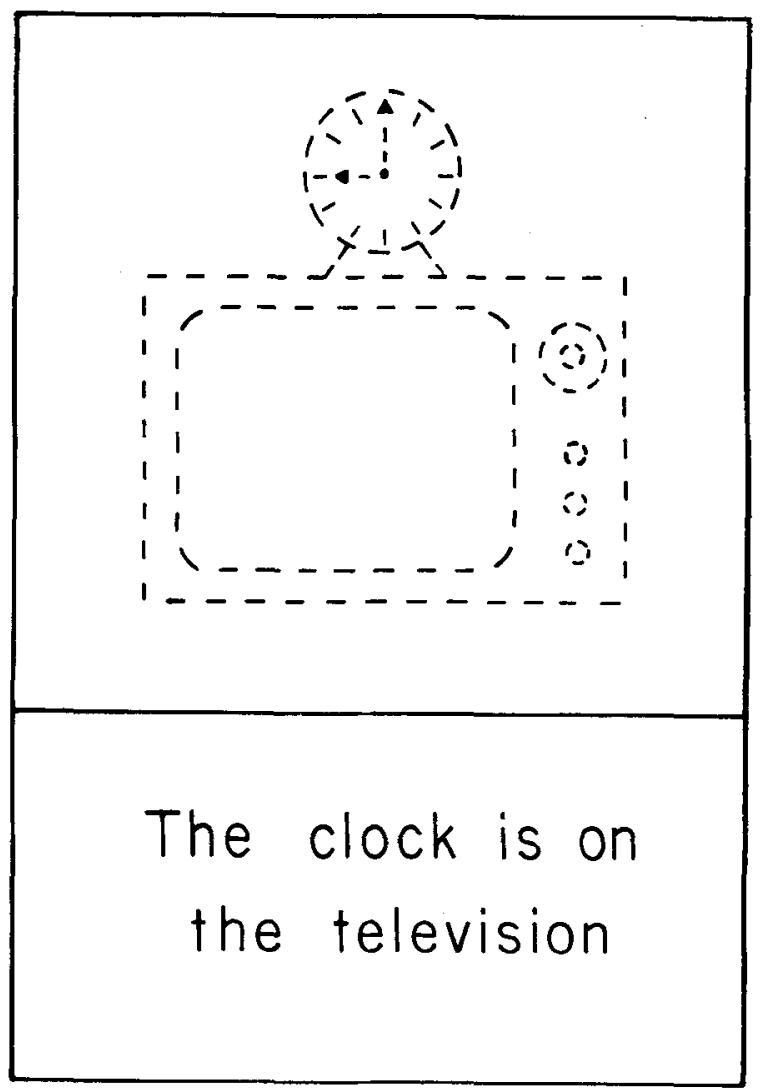

Figure 2. The relationship between the concepts "clock" and "television" expressed as a schematic diagram and as a sentence. 
digital computer. Each subject was seated in a dimly lit, individual cubicle directly facing a BWD 594 cathode-ray oscilloscope with P31 phosphor. A videomonitor and headphones for subjectexperimenter communication were also included in each cubicle. The response buttons were placed $1 \mathrm{~cm}$ apart at armrest level in front of the oscilloscope.

\section{Results}

Mean reaction time and accuracy values were calculated for the unique- and shared-concepts conditions in each trial block in which a subject attained an overall accuracy score of at least $85 \%$. The block means for each concept status condition were averaged for each subject to provide the individual scores for subsequent analyses. All subjects reached the $85 \%$ criterion in four or more blocks.

The results are summarized in Table 2 , in which it is clear that concept status affected performance in the sentence condition only. The speed and accuracy data were complementary, and there was no suggestion of a tradeoff between them in the relevant conditions. An analysis of variance conducted on the individual scores showed that the interaction between modality and concept status was significant $[\mathrm{F}(1,24)=50.71$, MSe $=$ $3,063, p<.01]$ in the reaction time data. A planned comparison showed that the effect of concept status was significant in the sentence condition $[\mathrm{t}(12)=7.56]$. The direction of the difference in the picture condition was contrary to that predicted by the model. A twotailed test indicated the difference to be significant at $\alpha=.05[t(12)=1.90]$. There were no significant main effects in the accuracy data, but the interaction between modality and concept status was significant $[F(1,24)=$ $16.08, \mathrm{MSe}=16.29, \mathrm{p}<.01 \mathrm{]}$. The false alarm rates were $7.2 \%$ and $7.0 \%$ in the sentence and picture conditions, respectively.

\section{Discussion}

HAM's prediction that it takes longer to retrieve information about sentences with shared concepts than about sentences with unique concepts was confirmed. In addition to providing a general replication of Anderson (1974), this result extends the effect to stimuli that express simple and familiar semantic relationships. As

Table 2

Mean Reaction Time, Variance, and Accuracy as a Function of Stimulus Class and Concept Status in Experiment 1

\begin{tabular}{ccrrr}
\hline $\begin{array}{c}\text { Stimulus } \\
\text { Class }\end{array}$ & $\begin{array}{c}\text { Concept } \\
\text { Status }\end{array}$ & \multicolumn{1}{c}{ RT } & Variance & Accuracy \\
\hline \multirow{2}{*}{ Sentences } & Unique & 875 & 41,503 & 97.1 \\
& Shared & 1,080 & 123,912 & 92.0 \\
\multirow{2}{*}{ Pictures } & Unique & 865 & 47,724 & 92.6 \\
& Shared & 831 & 76,618 & 96.5 \\
\hline
\end{tabular}

Note-Mean reaction time is given in milliseconds; variance is given in square milliseconds; accuracy is expressed as percent correct. the learning procedure used in Experiment 1 also differed markedly from that used by Anderson, our data suggest that HAM's prediction for sentences may generalize to a variety of different experimental conditions.

HAM's prediction that retrieval of pictures with unique concepts will be faster than retrieval of pictures with shared concepts was not confirmed. Although the experimental procedure for pictures was identical to that for sentences, no increase in reaction time was found for pictures with shared concepts. A significant difference did exist, but it was in the nonpredicted direction.

It is difficult to conceive how the present form of HAM can accommodate such findings. HAM can be divided into three independent stages: the parsing stage, the search-and-match stage, and the response execution stage. In the parsing stage, pictures and sentences are processed by perceptual and linguistic parsers, respectively, until they form similar tree structures. It is likely that one parser may process its input material faster than the other, thus adding a longer processing time to one stimulus class, or that both parsers process simple, familiar material faster than complex, novel material. To explain the results for pictures, however, it must be assumed that the perceptual parser processes pictures with unique concepts slower than pictures with shared concepts in a way that negates the faster search and retrieval time of the unique-concepts condition in the next stage. There is no obvious theoretical reason for such a mechanism; however, in the present study, subjects received more practice with the schematic stimuli in the shared condition than in the unique condition, and this could have facilitated encoding. Consideration of the block data does not support such an interpreta. tion. There was no consistent interaction between concept status and practice in the pictorial condition. If the absence of a fan effect under these conditions is due to the effect of differential practice on the encod. ing stage, it must be assumed that the benefit is achieved in one block of trials and is unaffected by subsequent familiarization with the stimuli.

The search-and-match stage forms the section of HAM that has been outlined in most detail. It is from this stage that the predictions tested in this experiment were drawn. Sentence- and picture-derived tree structures are assumed to be stored in the same format in a common long-term memory store. All tree structures in this store are subject to the same search procedure when a tree from working memory is matched with a tree in long-term memory. This stage cannot account for the results obtained for pictures unless it is assumed that the search procedures operating on picture propositions are different from those operating on sentence propositions. While the sentence-derived propositional trees would still be searched in the serial manner specified by Anderson and Bower (1973), the picture-derived propositional trees would need to be searched in a parallel form similar to that specified for sentences by Quillian 
(1968). Such a modification represents a radical change in the nature of HAM, however.

The response execution stage is embodied in the executive and language generation sections of HAM. To account for the results for pictures, this stage must negate the faster retrieval of pictures with unique concepts in a similar manner to that postulated for the parsing stage. As positive and negative responses are decided in the search-and-match stage, contingent upon whether a match does or does not occur, the response requires only organization in a form suitable for output. It is unlikely that this stage is affected by the nature of the concepts in pictures but not in sentences or even that it is affected by stimulus class at all. In summary, then, it seems unlikely that the present form of HAM or any simple modification thereof can parsimoniously account for the results obtained for pictorial material in Experiment 1.

\section{EXPERIMENT 2}

Experiment 2 was designed to replicate and extend the results of the first experiment. The basic design was similar in that it included the same modality (sentences/ pictures) and concept status (unique/shared) conditions, but two important modifications were made. First, modality was treated as a within-subjects factor. This modification was included in order to assess the stability of the observed differences between picture and sentence recognition. Do these differences reflect fixed properties of the representation and retrieval systems involved with the two modalities, or do they result from selection of a particular storage or processing mode in a given stimulus environment? If the first of these positions is correct, then the basic relationship between stimulus classes should be observed again in Experiment 2. However, if optional processes are involved in either acquisition or retrieval, it is possible that the relationship will be altered by application of a withinsubjects design.

The second modification involved an additional concept status condition. In addition to the intramodality conditions used in the first experiment, Experiment 2 included intermodality conditions involving shared concepts. In these conditions, stimuli were always learned and tested in the same modality, but they shared each of their concepts with stimuli in the alternative modality. For example, if "The clock is on the television" is learned and tested in pictorial form, the item (clock) and the object (television) would each be included, in separate stimuli, in the sentence set. This manipulation permits direct consideration of the effect of propositions established in one modality on reaction time for stimulus combinations in the alternative modality.

Consideration of the results from Experiment 1 leads directly to an asymmetric prediction for the inter- modality conditions in Experiment 2. If additional propositions established in the same modality have no effect on reaction time for pictures, it seems unlikely that additional propositions derived from sentences will produce a different result. Performance in the sentence condition may be more interesting, however. If pictorial stimuli are (also) represented in the format pertaining to sentences, it seems likely that reaction time in the relevant intermodality condition will be increased relative to the unique-concepts condition.

\section{Method}

Design. Except for the addition of the intermodality conceptsharing conditions within each modality and the use of repeated observations on all factors, the overall design followed Experiment 1 . There were two modalities, sentences and pictures, old and new stimulus combinations within each modality, and, nested in the "old" condition, a unique-concepts condition, a shared intramodality condition, and a shared intermodality condition.

Every subject completed eight blocks of trials in the single experimental session. Each block consisted of 24 sentences followed by 24 pictures, with an equal distribution of old and new stimuli in each sequence of 24 trials. Of the 12 old stimuli in each modality, 4 had unique concept combinations, 4 shared their concepts with other stimuli from the same modality, and 4 shared their concepts with other stimuli from the alternative modality. The new stimuli were randomly constructed from the remaining concept combinations.

Eight blocks of 48 trials provided a total of 384 trials per subject. Only the responses to learned stimuli from those blocks in which subjects achieved at least $85 \%$ correct responses were analyzed.

Stimuli. The stimuli were drawn from the same total set of 225 item-object combinations that was used in Experiment 1. Both picture and sentence stimuli were displayed in the same form as before.

Stimulus selection. Stimulus selection followed Experiment 1 , to ensure that the subjects in different sessions learned different sets of stimuli. Before each session, a program randomly selected a fresh set of concept combinations for each condition. In this process, four items and four objects were allocated to the unique conditions in each modality, and two items and two objects were allocated to each of the three sharedconcepts conditions. The items and objects in the unique conditions were combined to form four stimuli with no shared relationships in each modality. The items and objects within each of the shared conditions were combined orthogonally $\left(\mathrm{i}^{1} \mathrm{o}^{1}\right.$, $\mathrm{i}^{1} \mathrm{o}^{2}, \mathrm{i}^{2} \mathrm{o}^{1}, \mathrm{i}^{2} \mathrm{o}^{2}$, where $\mathrm{i}$ and $\mathrm{o}$ represent items and objects, respectively) to form four stimuli in which each item was related to two objects and vice versa. New stimuli were formed from the remaining combinations of items and objects.

Procedure. The experimental procedure followed Experiment 1 , with a learning phase followed by a test phase that included a block of 48 trials, trial-by-trial feedback, and a further display of the target set. As in Experiment 1, the various concept subsets were blocked during the learning phase to facilitate acquisition.

Subjects. Nine males and eight females who had not participated in Experiment 1 were voluntary subjects in Experiment 2. All subjects attended the University of Western Australia and were between 18 and 36 years of age.

\section{Results}

Following the procedure used in Experiment 1, mean reaction times and percentage correct scores were 
calculated for each subject for each of the six experimental conditions. Dunn's procedure (Kirk, 1968) was then used to evaluate the two planned comparisons between sentence means and the two planned comparisons between picture means. The difference that a sentence or picture comparison had to exceed to be significant at the .05 alpha level was based on the pooled error variances for each of the three sentence or picture conditions, respectively. In each case, the degrees of freedom were 32. The sentence mean comparisons were evaluated by a one-tailed test, and the picture mean comparisons were evaluated by a two-tailed test.

Table 3 shows that mean reaction time for sentences with unique concepts was $42 \mathrm{msec}$ faster than for sentences with shared intramodality concepts and $125 \mathrm{msec}$ faster than for sentences with shared intermodality concepts. These differences were both significant; however, the difference between the unique and shared intramodality conditions is qualified by the presence of a possible speed-accuracy tradeoff. Although there were no significant differences in accuracy among the three conditions, this does not automatically refute an interpretation of the difference based on a change in the speed-accuracy criterion (Pachella, 1974). The planned comparisons between the unique- and shared-concepts conditions for pictures were not significant.

\section{GENERAL DISCUSSION}

Although the results from comparable conditions in the two experiments are broadly similar, substantial qualification is required. First, where sentences are concerned, the effect of an additional proposition is dramatically less in Experiment 2. The actual figures are $205 \mathrm{msec}$ and $42 \mathrm{msec}$ in Experiments 1 and 2, respectively. Second, although the difference in Experiment 2 is significant, it is qualified by the high error rate in the unique-concepts condition. Thus, although the results from both experiments are consistent with those from previous studies that have varied proposition set size (Anderson, 1974; Anderson \& Hastie, 1974; Lewis \&

Table 3

Mean Reaction Time, Variance, and Accuracy as a Function of Stimulus Class and Concept Status in Experiment 2

\begin{tabular}{crrrr}
\hline \multicolumn{2}{c}{ Concept Status } & RT & Variance & Accuracy \\
\hline \multirow{4}{*}{ Unique } & \multicolumn{4}{c}{ Sentences } \\
Shared & 980 & 44,251 & 92.6 \\
& Intramodality & 1,022 & 89,377 & 95.1 \\
& Intermodality & 1,105 & 47,150 & 88.9 \\
Unique & \multicolumn{4}{c}{ Pictures } \\
Shared & Intramodality & 791 & 50,507 & 98.0 \\
& Intermodality & 791 & 18,087 & 98.0 \\
\hline
\end{tabular}

Note-Mean reaction time is given in milliseconds: variance is given in square milliseconds; accuracy is expressed as percent correct.
Anderson, 1976), they raise the possibility that the presence of pictorial stimuli in some trials influences the representation or retrieval of information about sentences. This hypothesis clearly requires further evaluation.

The results for pictures are virtually identical in the two experiments. In both cases, mean reaction time was slightly faster in the shared-concepts condition than in the unique-concepts condition. Considered together, then, the results from the intramodality conditions in the two experiments lend no support to the view that information about sentences and pictures depends on common representational and retrieval systems. On the contrary, they suggest that one or both of these systems are qualitatively different in the two cases. Is it possible to retain the representational component advocated by Anderson and Bower (1973) by assuming that qualitatively distinct retrieval processes are invoked for sentences and pictures? Perhaps, but just how this would operate in the original model is not clear. If retrieval is contingent on completion of the parsing stage, and if the outputs from the perceptual and sentence parsers are identical during both acquisition and retrieval, there appears to be no scope in Anderson and Bower's model for further, modality-specific processing. Thus, the representational and retrieval components are interdependent with regard to modality, and it is not possible to argue, for example, that serial and parallel retrieval processes are invoked for sentences and pictures, respectively. In more recent formulations (Anderson, 1976; Anderson \& Paulson, 1978), modality-specific subnodes are included, and it is therefore possible to retain one component of the model. For example, the propositional description of representation can be retained if it is assumed that qualitatively distinct retrieval processes are invoked for sentences and pictures.

So far as pictorial stimuli are concerned, the results of our experiments are clearly inconsistent with those reported by Anderson and Paulson (1978) from an experiment in which they used selected facial features to manipulate concept status. Why? Perhaps the weakest link in our experiments is that subjects received differential practice with the schematic stimuli in the unique and shared intramodality conditions during learning. That such an influence may be present is supported by the small advantage found for the shared intramodality condition in both experiments for pictures. The overall results of Experiment 2 are not consistent with this interpretation, however. If the effect were due to facilitation in an encoding or parsing stage, reaction time in the shared intramodality condition should be shorter than reaction time in the shared intermodality condition as well, but performance in these two conditions was identical. This problem clearly demands further investigation under conditions in which differential practice is not a confounding factor.

An alternative explanation involves differences in the nature of the stimuli used in the two studies. In the 
present study, the propositions shared between pictures were constructed from sets of independent elements that can be and often are combined in one place in the world. The stimuli used by Anderson and Paulson (1978), on the other hand, included elements that normally enjoy a mutually exclusive relationship. Assuming that the extent to which information from separate displays can be integrated in memory is influenced by experience, it is suggested that the pictorial stimuli used in our studies encouraged the use of integrated mental representations, whereas the stimuli used by Anderson and Paulson (1978) precluded this, and that such integration facilitates subsequent recognition.

Essentially the same point has been made by Moeser (1979). She found that the fan effect occurred only when repeated concepts are stored as independent episodes. When interrelated sentences were presented in a common block, performance was actually facilitated by concept repetition. From this evidence, Moeser concluded that the fan effect reflects interference arising from searching a set of independently stored sentences that share common concepts, not as a result of interference from searching a network of interconnected sentences. Our contention is that Anderson and Paulson's (1978) stimuli could only be treated as independent episodes and that the fan effect observed by them reflects a search through these episodes in memory. The pictorial stimuli used in our study encouraged the use of integrated storage representations, and recognition efficiency benefited accordingly. Further support for the view that a common process may be involved in our pictorial conditions and in Moeser's (1979) integrated storage conditions may be derived from the fact that in both cases a reverse fan effect was obtained.

One implication of this interpretation is that the question of representational equivalence remains open. Pictorial and verbal representation of overlapping concept combinations may produce quite different fan and retention effects because of the effect of modality on interstimulus integration, not because of intrinsic differences in representation.
The intermodality results from Experiment 2 are clear-cut and in no way qualified by differential practice with the pictorial stimuli or a speed-accuracy tradeoff. At a descriptive level, they indicate that, whereas the representation involved in sentence memory is influenced by pictorial stimuli, the reverse does not apply. Here, too, the present data do not permit us to discriminate between explanations based on acquisitional, representational, and retrieval differences. They do, however, lend direct support to the conclusion that the processes involved in long-term memory for pictures and sentences are not qualitatively identical.

\section{REFERENCES}

Anderson, J. R. Language, memory and thought. Hillsdale, N.J: Erlbaum, 1976.

Anderson, J. R. Retrieval of propositional information from long-term memory. Cognitive Psychology, 1974, 6, 451-474.

Anderson, J. R., \& Bower, G. H. Human associative memory. Washington, D.C: Winston, 1973.

Anderson, J. R., \& Hastie, R. Individuation and reference in memory: Proper names and definite descriptions. Cognitive Psychology, 1974, 6, 495-514.

Anderson, J. R., \& Paulson, R. Interference in memory for pictorial information. Cognitive Psychology, 1978, 10, 178-202.

KIRK, R. E. Experimental design: Procedures for the behavioural sciences.Belmont, Calif: Brooks/Cole, 1968.

LEwIS, C. H., \& ANDERson, J. R. Interference with real world knowledge. Cognitive Psychology, 1976, 8, 311-335.

Moeser, S. D. The role of experimental design in investigations of the fan effect. Journal of Experimental Psychology: Human Learning and Memory, 1979, 5, 125-134.

Pachella, R. G. An interpretation of reaction time in information processing research. In B. Kantowitz (Ed.), Human information processing: Tutorials in performance and cognition. New York: Erlbaum, 1974.

PAIvio, A. Images, propositions, and knowledge. In J. M. Nicholas (Ed.), Images, perception and knowledge. Dordrecht, The Netherlands: Reidel, 1976.

Quillian, M. R. Semantic memory. In M. Minsky (Ed.), Semantic information processing. Cambridge, Mass: M.I.T. Press, 1968.

(Received for publication October 22, 1979; revision accepted June 23,1980 .) 